\title{
MAPEAMENTO DA PRODUÇÃO CIENTÍFICA SOBRE INCERTEZA AMBIENTAL EM BASES INTERNACIONAIS
}

Data de submissão: 14/06/2014 Aceite: 10/06/2016

Elvis Silveira-Martins ${ }^{1}$

Carlos Ricardo Rossetto ${ }^{2}$

\section{RESUMO}

O objetivo deste artigo é mapear a produção científica sobre o tema incerteza ambiental nas bases de dados internacionais ProQuest e EBSCO, fornecendo resultados sobre o assunto, bem como as características dos trabalhos científicos já publicados. O método utilizado foi a bibliometria, sendo investigados 97 artigos de um universo de 1.194. Os trabalhos analisados compreenderam o período de 1975 a 2010. Os resultados sugerem que: a) durante o período analisado, diversas redes sociais de pesquisadores e instituições se formaram para estudar esse tema; b) o trabalho de Ducan (1972), embora tenham se passado quase quatro décadas da sua publicação, continua influenciando a formulação de variáveis para a mensuração da incerteza ambiental; e c) a pesquisa sobre incerteza ambiental recebeu, no decorrer dos anos, outras terminologias e metodologias. Observou-se que os estudos foram aplicados nos mais diversos segmentos empresariais, sempre objetivando minimizar as turbulências ambientais e com isso maximizar os resultados organizacionais. Esta pesquisa revelou, ainda, que a forma para a sua mensuração não pode ser normatizada, visto que cada segmento se depara com um ambiente diferente e com variáveis diversas (clientes, fornecedores, concorrentes, sociedade, política, ações governamentais, entre outras).

Palavras-chave: Incerteza ambiental; Ambiente; Bibliometria.

\footnotetext{
1 Possui graduação em Administração de Empresas pela Universidade de Passo Fundo, UPF, mestrado em Administração pela Universidade do Vale do Itajaí, UNIVALIA, doutorado em Administração e Turismo pela Universidade do Vale do Itajaí, UNIVALI. Pelotas, RS-Brasil. E-mail: elvis.professor@gmail.com 2 Possui graduação em Engenharia Civil pela Universidade Católica de Pelotas, UCPE, mestrado em Engenharia de Produção pela Universidade Federal de Santa Catarina, UFSC, doutorado em Engenharia de Produção pela Universidade Federal de Santa Catarina, UFSC. Biguaçu, SC - Brasil E-mail: rossetto@ univali.br
} 


\section{INTRODUÇÃO}

Empresas dos mais variados tamanhos e segmentos são submetidas diariamente ao dinamismo do mercado, fruto de acirrada concorrência que acaba por forçar os gestores a tomarem decisões em tempo real com precisão cirúrgica, sob pena de prejuízos, muitas vezes irreversíveis. No entender de Wallace et al. (2010), ambientes estáveis são caracterizados por alterações mínimas nas preferências dos clientes, tecnologias e dinâmica competitiva, enquanto setores altamente dinâmicos são caracterizados por uma elevada taxa de mudança e instabilidade, aumentando a incerteza na decisão. No entanto, todas as empresas estão, involuntariamente, sucetíveis às condições ambientais.

Para o correto entendimento de situações vivenciadas pelas organizações em decorrência de incertezas ambientais, a moderna gestão exige uma interpretação detalhada, por meio das variáveis específicas que circundam a empresa, o que poderá resultar em um desempenho superior ao da concorrência. Miller (1992) enfatiza que as variáveis ambientais não compreendem somente as externas à organização, no seu entender deve-se levar em consideração o ambiente interno nas tomadas de decisões. Ainda segundo o autor, um desequilíbrio duradouro na organização, com relação à estrutura ou processo, pode vir a resultar na busca incessante de ajustes internos, em que a incerteza manifesta-se como desafio nas tomadas de decisões do corpo gerencial, que devem espelhar os melhores intérpretes da nova tecnologia emergente e novos mercados competitivos.

Dessa forma, perceber as incertezas proporcionadas pelo ambiente torna-se fundamental para a performance do empreendimento. Essa dicotomia (incerteza ambiental e performance) é pesquisada há mais de três décadas, a exemplo dos trabalhos de Huber, O'Connell e Cummings (1975), Weed e Mitchell (1980), Bourgeois III (1985), Stearns, Hoffman e Heide (1987), Koberg e Ungson (1987), Swamidass e Newell (1987), Shrader, Mulford e Blackburn (1989), Boyd (1990), Chow e Haddad (1991), Gul (1991), Gerloff, Muir e Bodensteiner (1991), Kren e Kerr (1993), Germain, Dröge e Daugherty (1994), Tan e Litschert (1994), Sabherwal e Kirs (1994), Reed, Lemark e Montgomery (1996), Chong e Chong (1997), Kumar e Seth (1998), Abramson e Ai (1998), Luo (1999), Vickery, Calantone e Dröge (1999), Claycomb, Dröge e Germain (2001), Waldman et al. (2001), Sarkar, Echambadi e Harrison (2001), Li e Atuahene-Gima (2002), Kreiser e Marino (2002), Gosselin (2005), Desarbo et al. (2005), Lee, Lin e Pai (2005), Babakus, Yavas e Haahti (2006), Fink, Edelman e Hatten (2006), Carmeli e Tishler (2006), Agle et al. (2006), Krishnan, Martin e Noorderhaven (2006), Paulraj e Chen (2007), Liao e Tu (2007), Cannella Jr., Parke e Lee (2008), Sun, Hsu e Hwang (2009), Wallace et al. (2010), Silveira-Martins e Tavares (2014a, 2014b).

Diante desses aspectos, observa-se a importância de se discutir e entender esse tema que desperta o interesse de pesquisadores de estratégia e gestores. Nessa perspectiva, o presente estudo objetiva mapear a produção científica sobre o tema incerteza ambiental nas seguintes bases de dados internacionais: ProQuest e EBSCO.

Miles, Snow e Pfeffer (1974) já demonstram a importância de entender as variáveis ambientais, em especial as incertezas produzidas por elas. Segundo os autores, um número crescente de estudos tem sido desenvolvido, no entanto, na melhor das hipóteses, com resultados modestos, gerando confusões sobre o papel da organização em contrapartida às demandas ambientais, além das suas ligações com a tecnologia, estrutura e processos organizacionais. Dessa maneira, os autores justificam o desenvolvimento de pesquisas com contribuições à temática, uma vez que as inconstâncias geradas pelas incertezas ambientais afligem todas as áreas relacionadas ao comportamento organizacional. 
Ao abordar a incerteza ambiental sob a luz da ecologia populacional, Hannan e Freeman (1977) destacam que a interpretação dos efeitos do ambiente sobre a estrutura organizacional mudou-se para um lugar central na teoria e pesquisa das organizações nos últimos anos. Essa mudança abriu uma série de possibilidades que motivam o desenvolvimento de pesquisas sobre o tema. Em complemento, Simon (1978) afirma que muitos dos pontos fracos organizacionais devem-se, em grande parte, ao não conhecimento de todas as alternativas, à incerteza sobre eventos exógenos relevantes e à incapacidade de calcular consequências. Assim, as tomadas de decisões em condições de incerteza são consideradas uma das habilidades mais importantes exigidas dos tomadores de decisões (SIMON, 1991).

Diante desse contexto, observa-se a importância do desenvolvimento de pesquisas que abarquem a temática incerteza ambiental, procurando verificar as suas abordagens, seja pela ótica do comportamento do gestor (MILES; SNOW; PFEFFER, 1974), da ecologia populacional (HANNAN; FREEMAN, 1977), da racionalidade nas tomadas de decisões (SIMON, 1978, 1991), seja por outros prismas que poderão nortear gestores e pesquisadores interessados nos conhecimentos produzidos sobre esse construto.

Dessa maneira, justifica-se o presente estudo pela necessidade de explorar a temática incerteza ambiental em produções científicas reconhecidas pela academia, verificando suas características em pesquisas internacionais e, com isso, subsidiando novas pesquisas. Outro aspecto que legitima esta pesquisa é o fato de não terem sido encontrados estudos similares com esta abordagem no Brasil, além de Silveira-Martins et al. (2013), que abordaram a temática incerteza ambiental no âmbito nacional.

Nesse sentido, este artigo está organizado em cinco seções. A primeira parte explora, passo a passo, a gênese, o desenvolvimento e a consolidação da temática incerteza ambiental. Na segunda seção, apresenta-se o quadro teórico de referência que procura situar o contexto intelectual em que se empreendeu o estudo; na sequência, aborda-se a metodologia que orientou a condução do estudo sob análise. Na quarta seção, discute-se e pondera-se sobre a análise dos dados bibliográficos e, na última seção, à guisa de conclusão, tecem-se reflexões e novas direções e possibilidades para estudos sobre incerteza ambiental.

\section{INCERTEZA AMBIENTAL}

A definição do conceito de incerteza ambiental é permeada por três componentes: i) a falta de informação sobre os fatores ambientais associados a uma determinada situação de tomada de decisão; ii) o desconhecimento do resultado de uma decisão específica em termos de quanto a organização poderá perder se a decisão for incorreta; e iii) a incapacidade de atribuir probabilidades com algum grau de confiança no que diz respeito à forma como os fatores ambientais irão afetar o sucesso ou insucesso da unidade durante a gestão do tomador de decisão (DUCAN, 1972).

O autor estudou decisões de grupos em indústrias, objetivando identificar as características do ambiente que contribuem para que os membros das unidades de decisão possam gerenciar as incertezas ambientais. No estudo, duas dimensões são identificadas: a) dimensão simples/ complexo, definida como o número de fatores levados em consideração nas tomadas de decisão; e b) dimensão estático/dinâmico, entendida como o grau em que esses fatores no ambiente da unidade de decisão continuam a ser basicamente os mesmos ao longo do tempo, ou estão em um processo contínuo de mudança. Os resultados indicaram que os indivíduos em unidades de decisão em ambientes dinâmicos/complexos experimentam a maior quantidade de incerteza no processo decisório. Os dados também indicam que a dimensão estático/dinâmico do ambiente contribui mais para a incerteza do que a dimensão simples/complexo. 
Kreiser e Marino (2002), após analisarem sistematicamente o desenvolvimento histórico da incerteza, concluíram que múltiplas operacionalizações têm sido desenvolvidas ao longo dos últimos 60 anos para medir a quantidade de incerteza presente no ambiente externo. Cada uma dessas medidas pode ser efetivamente utilizada na pesquisa de desempenho organizacional, dependendo das questões específicas de investigação a serem abordadas. As conceituações de incerteza continuaram a evoluir e divergem entre si ao longo dos últimos 60 anos, integrando linhas de pesquisa, o que impede a generalização dos resultados sobre este tema.

A maioria das decisões econômicas é complexa e muitas vezes precisa de informações de mais de uma fonte. Assim, os gestores de pequenos empreendimentos empresariais, buscando expandir e/ou manter o empreendimento, precisam saber sobre fatores como disponibilidade de recursos humanos, financiamento e potencial de retaliação dos concorrentes. Enquanto os gestores de pequenos empreendimentos poderiam pesquisar essas questões sozinhos, muitas vezes é mais barato e conveniente obter informação e conselhos de outras pessoas. Em outras palavras, é importante desenvolver uma rede de contatos pessoais, já que tais redes podem desempenhar um papel vital na redução da incerteza, facilitando o recebimento de informações. Nesse sentido, é importante destacar outro aspecto importante: um networking eficaz pode consumir uma quantidade considerável de tempo e energia, especialmente para empresários e gestores de novos empreendimentos, o que poderá ocasionar mais problemas do que soluções organizacionais. Esses foram os achados de um estudo desenvolvido por McGee e Sawyerr (2003) que procurou analisar a relação entre a percepção estratégica, a análise ambiental e as fontes de informação utilizadas pelos proprietários e gerentes de pequenas indústrias de alta tecnologia.

Mais recentemente, a pesquisa de Semadeni e Anderson (2010) objetivou avaliar organizações e as características que influenciam no nível de imitação (de produtos ou serviços do concorrente) sob condições de alta incerteza ambiental e assimetria de informação. Os resultados apontaram para a interação entre essas características organizacionais e o nível de imitação. No entanto, os resultados não são conclusivos, visto que essa análise depende das particularidades da organização e dos fatores da oferta. Não obstante, mesmo a pesquisa não sendo ilativa, ela enriquece o conhecimento dos gestores para a tomada de decisão sobre imitar ou não produtos e serviços para superar as condições de incerteza ambiental.

Observa-se, no estudo desenvolvido por Wallace et al. (2010), o teste dos focos de regulamentação das pequenas empresas (foco de promoção e foco de prevenção) e sua correpondência com o desempenho da organização de maneira diferente quando os níveis de incerteza ambiental variam. Os resultados sugerem que o foco de promoção está positivamente relacionado com o desempenho da empresa, enquanto o foco de prevenção é negativamente relacionado ao desempenho da organização. É necessário observar que essas relações foram moderadas pelo grau de dinamismo ambiental. Em ambientes mais dinâmicos, a relação entre o foco de promoção e o desempenho da empresa é reforçada, enquanto a relação entre o foco de prevenção e o desempenho da empresa é afetada negativamente. $O$ inverso foi encontrado para ambientes menos dinâmicos.

\section{METODOLOGIA}

Os procedimentos metodológicos desta pesquisa estão ancorados na técnica da bibliometria. De acordo com Araujo et al. (2000), esse tipo de estudo busca observar a evolução da literatura e o conhecimento produzido no decorrer dos anos.

Nesses moldes, foram utilizados os termos Environmental Uncertainty e Environment Uncertainty para localizar artigos referentes à incerteza ambiental nas bases de dados ProQuest e EBSCO. 
Considerou-se que essas palavras-chave deveriam constar no título ou no resumo dos trabalhos. Foram desconsiderados todos os artigos que não se encontravam completos, além das críticas literárias. Esse procedimento resultou em um total de 402 artigos na base ProQuest e 792 na base EBSCO.

Na sequência, consideraram-se apenas os artigos que possuíam Index $H$ igual ou maior do que 5; após essa seleção, restaram 124 artigos originários da base ProQuest e 56 da EBSCO. 0 terceiro filtro aplicado foi o de exclusão de artigos que se encontravam nas duas bases e os que utilizavam os termos Environmental Uncertainty e Environment Uncertainty de maneira genérica, sem vínculo com a temática de estratégia. $O$ Quadro 1 sintetiza esse processo de seleção de artigos que resultou em um total de 97 artigos válidos, compreendendo o espaço temporal entre os anos de 1975 e 2010.

Quadro 1 - Síntese do processo de seleção de artigos

\begin{tabular}{|c|c|c|c|c|c|c|}
\hline \multicolumn{3}{|c|}{ PROQUEST } & \multicolumn{3}{c|}{ EBSCO } & \multirow{2}{*}{ TOTAL } \\
\hline Total de artigos & Alto impacto & Terceiro filtro & Total de artigos & Alto impacto & Terceiro filtro & \\
\hline 402 & 124 & 84 & 792 & 56 & 13 & 97 \\
\hline
\end{tabular}

Fonte: elaborado pelos autores (2014).

Após a apresentação dos aspectos metodológicos que nortearam o desenvolvimento desta pesquisa, a seguir será realizada a análise bibliométrica sobre as publicações identificadas na coleta dos dados. 


\section{ANÂLISE BIBLIOMÉTRICA}

Entre os artigos analisados, observou-se a formação de redes de cooperação, entre autores, para o desenvolvimento de pesquisas sobre incerteza ambiental. Nesse sentido, Dröge $(1986,1988,1994,1999,2001)$ recebe destaque pelo número de laços com seus pares, somando nove de entrada e nove de saída. Esses dados revelam que a autora, neste tema específico e nesta amostra de artigos, destaca-se pelas parcerias firmadas para o desenvolvimento de pesquisas científicas. Tais redes podem ser mais bem observadas na representação gráfica da Figura 1.

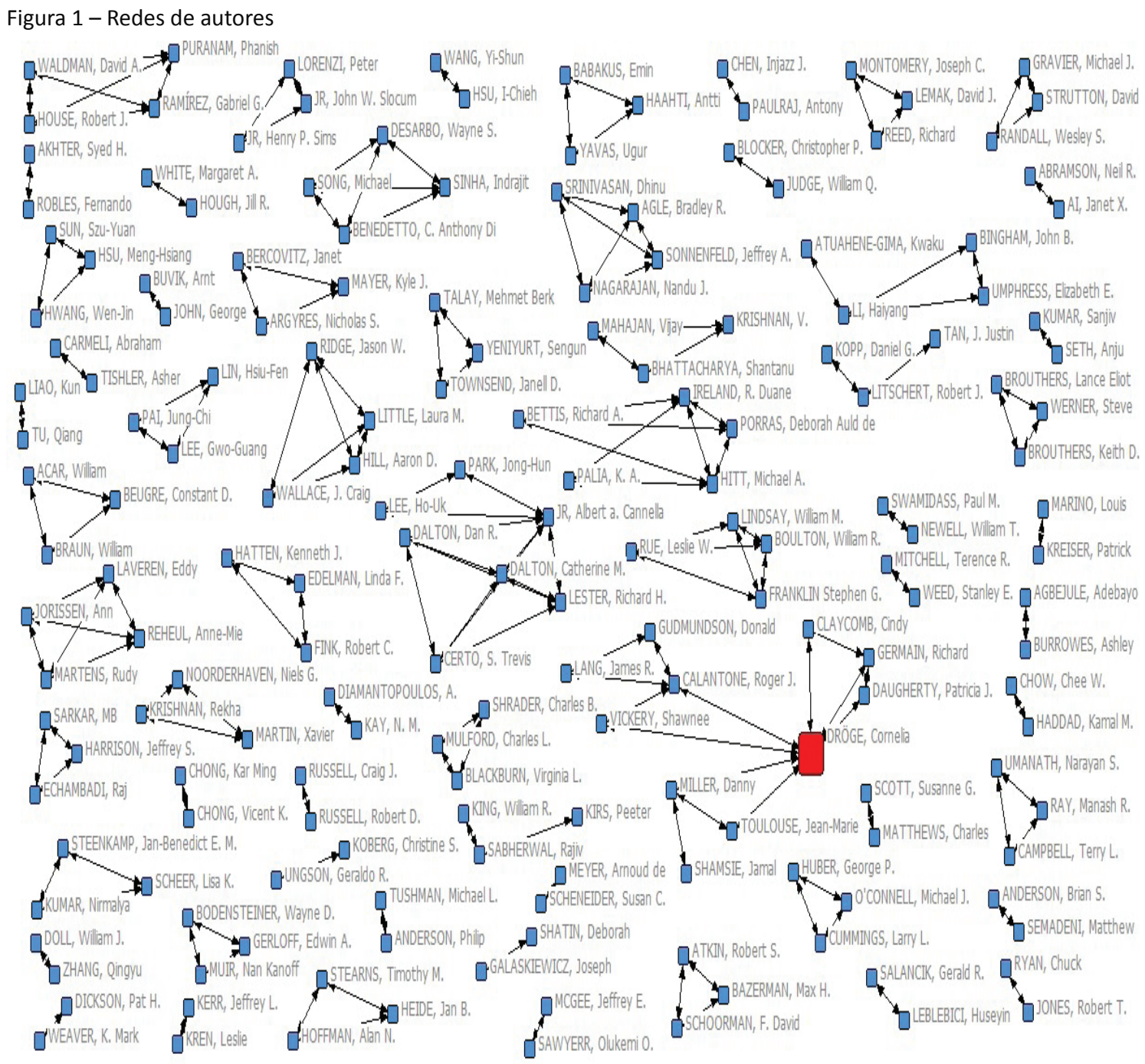

Fonte: elaborada pelos autores (2014).

As redes identificadas anteriormente não revelam, em regra, que os autores em destaque são os que mais produziram trabalhos sobre incerteza ambiental (apenas as redes de seus relacionamentos), no entanto, nesta pesquisa em específico, a exceção a essa regra deve ser aplicada. Dröge (1986, 1988, 1994, 1999, 2001), além de destaque em termos de redes de cooperação de autores, também é grafada como autora na maior quantidade de artigos publicados, somando um total de cinco artigos. $\mathrm{Na}$ sequência, aparecem Miller (1986, 1988, 1999), Litschert (1980, 1994), Hitt (1982, 1987), Ireland (1982, 1987), Koberg (1987), Sabherwal (1992, 1994), Sawyerr (1993, 2003), Germain (1994, 2001), Calantone 
$(1997,1999)$, Li $(2002,2007)$ e Cannella Jr. $(2006,2008)$, com dois trabalhos publicados cada um. Tornase importante ressaltar que não foram diferenciadas as ordens de assinatura de autoria dos trabalhos. Nos demais trabalhos analisados, os pesquisadores publicaram apenas um artigo.

Nessa mesma linha de raciocínio, buscou-se identificar a existência de redes de cooperação entre as instituições dos pesquisadores, assim diversas articulações foram caracterizadas. As instituições Texas A\&M University e Oklahoma State University, conforme a Figura 2, destacam-se das demais pelas parcerias que firmaram (laços de saída), num total de dez e nove, respectivamente.

Figura 2 - Redes de Instituições de Ensino Superior (IES)

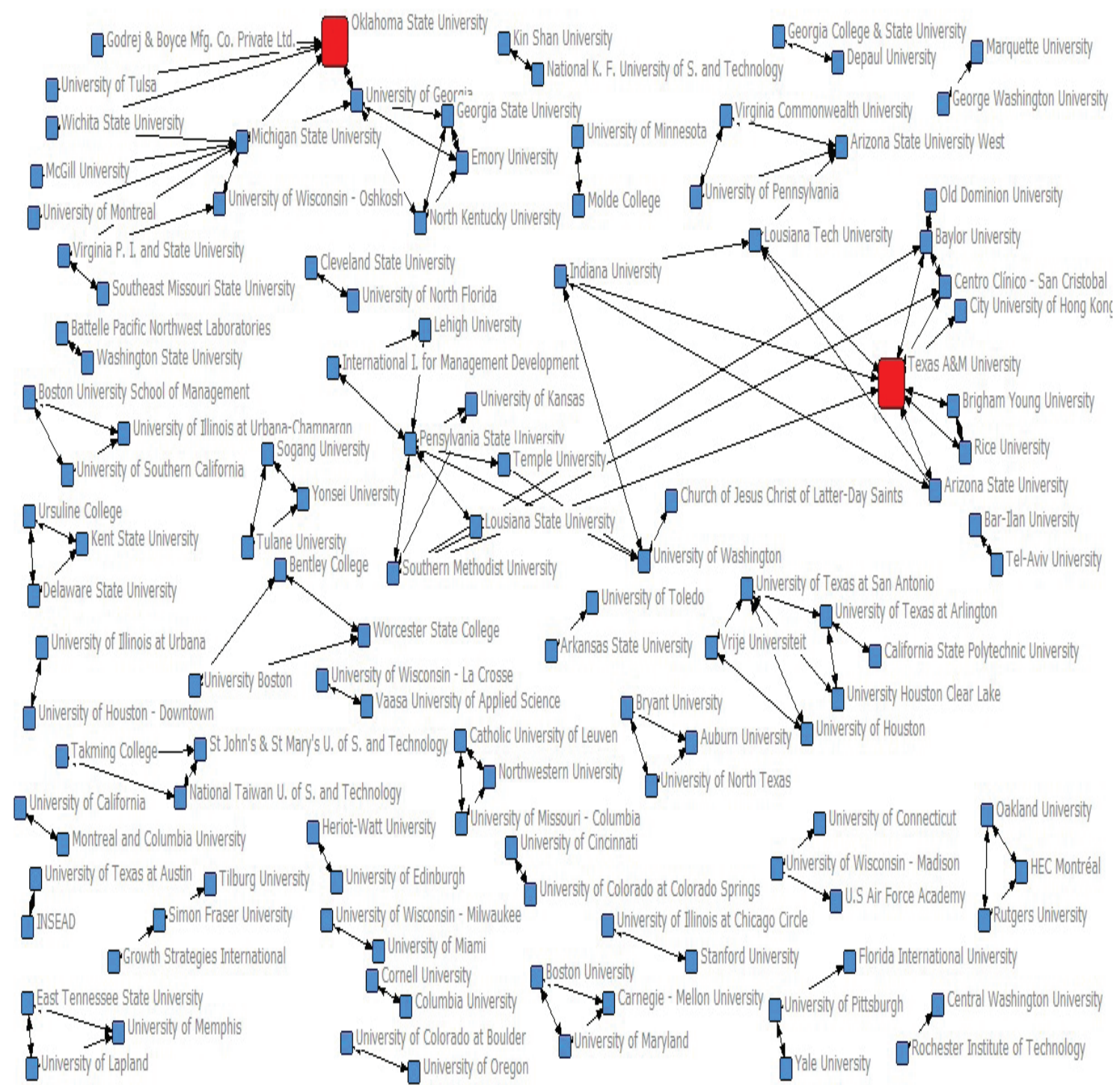

Fonte: elaborada pelos autores (2014).

Visando explorar não somente os autores e suas respectivas instituições, o Quadro 2 sintetiza os periódicos e o número de artigos neles publicados. Esse indicador busca auxiliar futuras submissões de trabalhos, pois identifica os periódicos que possivelmente possuam linhas de interesse em trabalhos que abordem o tema incerteza ambiental. Observou-se que, entre todos os estudados no periódico Academy of Management Journal, foram localizados 20 trabalhos entre os anos 1975 e 2010, 11 a mais do que o segundo com maior número de publicações, a saber, Strategic Management Journal. 
Quadro 2 - Periódico, índice $\mathrm{H}$ e quantidade de artigos publicados

\begin{tabular}{|c|c|c|}
\hline Título do periódico & Índice $\mathrm{H}$ & Artigos \\
\hline Academy of Management Journal & 119 & 20 \\
\hline Strategic Management Journal & 116 & 9 \\
\hline Journal of Management & 77 & 7 \\
\hline Journal of Small Business Management & 31 & 7 \\
\hline Administrative Science Quarterly & 86 & 4 \\
\hline Management Science & 97 & 4 \\
\hline Academy of Management Review & 114 & 3 \\
\hline Accounting and Business Research & 18 & 3 \\
\hline Decision Sciences & 45 & 3 \\
\hline Journal of International Business Studies & 74 & 3 \\
\hline Journal of Marketing & 99 & 3 \\
\hline Organization Science & 90 & 3 \\
\hline European Journal of Marketing & 19 & 2 \\
\hline International Journal of Manpower & 18 & 2 \\
\hline Journal of Knowledge Management & 16 & 2 \\
\hline Journal of Marketing Research & 70 & 2 \\
\hline Journal of Supply Chain Management & 13 & 2 \\
\hline Management Decision & 13 & 2 \\
\hline Supply Chain Management & 39 & 2 \\
\hline Business Process Management Journal & 13 & 1 \\
\hline European Business Review & 8 & 1 \\
\hline Europen Journal of Innovation Management & 9 & 1 \\
\hline Family Business Review & 13 & 1 \\
\hline International Journal of Productivity and Performance Management & 11 & 1 \\
\hline International Marketing Review & 29 & 1 \\
\hline Internet Research & 32 & 1 \\
\hline Journal of Global Information Management & 18 & 1 \\
\hline Journal of Manufacturing Technology Management & 21 & 1 \\
\hline Journal of Marketing Theory and Practice & 8 & 1 \\
\hline Management International Review & 12 & 1 \\
\hline Managerial Auditing Journal & 8 & 1 \\
\hline Managerial and Decision Economics & 14 & 1 \\
\hline Team Performance Management & 5 & 1 \\
\hline
\end{tabular}

Fonte: elaborado pelos autores (2014).

Na sequência, objetivando identificar a evolução no número de artigos publicados entre o período de 1975 e 2010, elaborou-se a Figura 3. Por meio desta, torna-se possível observar que, nos anos de 1987 e 2006, houve um aumento nas publicações, culminando no maior número de produções relacionadas ao tema incerteza ambiental, perfazendo um total de 16 pesquisas publicadas em periódicos de alto impacto.

A efetividade nesses anos se deve aos trabalhos de: Ireland et al. (1987), McCabe (1987), Milliken (1987), Stearns, Hoffman e Heide (1987), Koberg e Ungson (1987), Koberg (1987), Kay e Diamantopoulos (1987), Swamidass e Newell (1987), Babakus, Yavas e Haahti (2006), Lester et al. (2006), Akhter e Robles (2006), Fink, Edelman e Hatten (2006), Carmeli e Tishler (2006), Beugré, Acar e Braun (2006), Agle et al. (2006) e Krishnan, Martin e Noorderhaven (2006). 
Figura 3 - Produção científica por ano

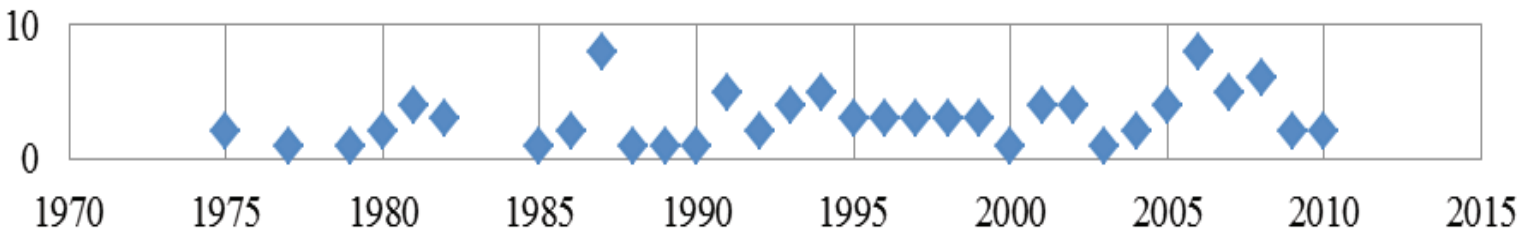

Fonte: elaborada pelos autores (2014).

Com o intuito de identificar como foi medida a incerteza ambiental e contribuir, assim, com futuras pesquisas, apresentam-se, no Quadro 3, os trabalhos analisados, os indicadores utilizados e as pesquisas que deram suporte à elaboração das variáveis. 
Quadro 3 - Variáveis utilizadas para mensuração da incerteza ambiental

\begin{tabular}{|c|c|c|}
\hline Artigo & Variáveis utilizadas & Trabalho de referência \\
\hline Huber, O'Connell e Cummings (1975) & $\begin{array}{l}\text { Operações militares; economia; negociações diplomáticas; atividades de } \\
\text { inteligência. }\end{array}$ & - \\
\hline Lev (1975) & Risco do estoque; risco das ações. & - \\
\hline Jones (1977) & Medidas de mudança; informação; previsão. & Ducan (1971) \\
\hline Sperkman (1979) & Compras fora da empresa; compras de outros departamentos. & Ducan (1971) \\
\hline Kopp e Litschert (1980) & Baixa e alta incerteza. & Ducan (1971); Downey et al. (1975) \\
\hline Weed e Mitchell (1980) & Informação e previsibilidade. & - \\
\hline Ford (1981) & Entidades internas à organização; entidades externas à organização. & Ducan (1971); Sathe (1974) \\
\hline Lorenzi, Jr., Jr. (1981) & Dinamismo; complexidade; imprevisibilidade. & Duncan (1972); Toni et al. (1973) \\
\hline Galaskiewicz e Shatin (1981) & $\begin{array}{l}\text { Estabilidade/instabilidade; homogeneidade/heterogeneidade; riqueza/ } \\
\text { pobreza. }\end{array}$ & Dill (1958); Thompson (1967); Aldrich (1972) \\
\hline Boulton et al. (1982) & Ambiente externo; medidas de incerteza; atividades de planejamento. & $\begin{array}{c}\text { Ducan (1972); Bourgeois (1978); Lindsay e Rue } \\
(1980)\end{array}$ \\
\hline Hitt, Ireland e Palia (1982) & $\begin{array}{l}\text { Relações com os fornecedores; preços dos concorrentes; qualidade e } \\
\text { mudanças de projeto; fornecedores financeiros; agências governamen- } \\
\text { tais; sindicatos. }\end{array}$ & Miles e Snow (1978) \\
\hline Leblebici e Salanik (1982) & Volatilidade dos preços no mercado. & - \\
\hline III (1985) & $\begin{array}{l}\text { Cliente; fornecedores; concorrente; sociopolítica; componente tecnoló- } \\
\text { gica. }\end{array}$ & Ducan (1972) \\
\hline Miller e Dröge (1986) & $\begin{array}{l}\text { Práticas mercadológicas; obsolescência de produtos; ações dos concor- } \\
\text { rentes; gastos de demanda e consumo; mudanças nos modos de produ- } \\
\text { ção e serviço. }\end{array}$ & Khandwalla $(1974,1977)$; Miller (1983) \\
\hline Tushman e Anderson (1986) & Previsão de erros da capacidade. & - \\
\hline Ireland et al. (1987) & $\begin{array}{l}\text { Fornecedores; concorrentes; clientes; finanças; agência reguladora; sin- } \\
\text { dicatos. }\end{array}$ & Miles e Snow (1978) \\
\hline McCabe (1987) & Informação; previsão de resultados; previsão de fatores ambientais. & Ducan (1972); Sathe (1974) \\
\hline Stearns, Hoffman e Heide (1987) & Publicidade. & - \\
\hline Swamidass e Newell (1987) & $\begin{array}{l}\text { Usuários; fornecedores; concorrentes; agências reguladoras; opiniões } \\
\text { políticas e públicas; sindicatos. }\end{array}$ & Duncan (1972); Bourgeois (1978) \\
\hline Boyd (1990) & Dinamismo ambiental; complexidade; munificência. & $\begin{array}{l}\text { Dess e Beard (1984); Keats e Hitt (1988); Gros- } \\
\text { sack (1965); Aldrich's (1979) }\end{array}$ \\
\hline Chow e Haddad (1991) & Alto; baixo. & \\
\hline
\end{tabular}




\begin{tabular}{|c|c|c|}
\hline Artigo & Variáveis utilizadas & Trabalho de referência \\
\hline Gul (1991) & $\begin{array}{l}\text { Concorrentes; tecnologia; produto/projeto; demanda; matéria-prima } \\
\text { (preço/disponibilidade); governo; sindicato. }\end{array}$ & $\begin{array}{c}\text { Miles e Snow (1978); Govindarajan (1984) } \\
-\end{array}$ \\
\hline Gerloff, Muir e Bodensteiner (1991) & Estado de incerteza; efeito de incerteza; resposta de incerteza. & Ducan (1972); Milliken (1987) \\
\hline Sabherwal e King (1992) & Dinamismo ambiental; heterogeneidade; hostilidade. & Friesen e Miller (1982) \\
\hline Sawyerr (1993) & Mudança no ambiente; complexidade ambiental; dependência do setor. & Daft et al. (1988) \\
\hline Kren e Kerr (1993) & $\begin{array}{l}\text { Clientes; concorrentes; fornecedores; grupos de regulação; tecnologias } \\
\text { industriais. }\end{array}$ & Govindarajan (1984) \\
\hline Miller (1993) & $\begin{array}{l}\text { Fornecedores; concorrentes; clientes; mercado de capitais/financeiros; } \\
\text { agências reguladoras; sindicatos. }\end{array}$ & $\begin{array}{c}\text { Miles e Snow (1978) } \\
-\end{array}$ \\
\hline Umanath, Ray e Campbell (1993) & № de produtos; no de áreas; $\mathrm{n}$ - de concorrentes; clima sociopolítico. & Ducan (1972) \\
\hline Germain, Dröge e Daugherty (1994) & $\begin{array}{l}\text { Práticas de comercialização; ações da concorrência; demanda e os gos- } \\
\text { tos do cliente; processos de produção. }\end{array}$ & - \\
\hline Tan e Litschert (1994) & Dinamismo; hostilidade; complexidade. & $\begin{array}{l}\text { Khandwalla (1977); Jauch, Osborn e Glueck } \\
\text { (1980) }\end{array}$ \\
\hline Sabherwal e Kirs (1994) & $\begin{array}{l}\text { Demanda; ações governamentais; disponibilidade do corpo docente; dis- } \\
\text { ponibilidade de pessoal/pessoal administrativo. }\end{array}$ & - \\
\hline Ganesan (1994) & Diversidade; volatilidade. & - \\
\hline Buchko (1994) & $\begin{array}{l}\text { Amplitude e frequência de troca de produtos de suas empresas e pro- } \\
\text { cessos. }\end{array}$ & Milliken (1987) \\
\hline Kumar, Scheer e Steenkamp (1995) & Tendências; volume da indústria; previsões de vendas. & Heide e John (1988) \\
\hline Matthews e Scott (1995) & $\begin{array}{l}\text { Clientes; fornecedores; distribuidores; concorrentes; governo; opinião } \\
\text { pública; tecnologia; mercados financeiros. }\end{array}$ & Ducan (1972); Jausch, Osborn e Glueck (1980) \\
\hline Mangaliso (1995) & $\begin{array}{l}\text { Educação, formação tecnológica e as habilidades dos funcionários; so- } \\
\text { cioculturais e o idioma da equipe; experiência tecnológica ou de gestão; } \\
\text { estilos gerenciais; mão de obra qualificada na organização; indivíduos e } \\
\text { grupos de integração; consumidores e/ou distribuidores; fornecedores, } \\
\text { equipamentos ou serviços; oferta de trabalho; concurso para fornecedo- } \\
\text { res ou clientes; governo; atitude do público com a empresa e seu produ- } \\
\text { to/serviço; sindicatos; necessidades tecnológicas. }\end{array}$ & Ducan (1972) \\
\hline Werner, Brouthers e Brouthers (1996) & $\begin{array}{l}\text { Políticas do governo; macroeconomia; recursos e serviços utilizados pela } \\
\text { empresa; mercado do produto e da procura; concorrência; incerteza da } \\
\text { tecnologia. }\end{array}$ & Miller (1993) \\
\hline
\end{tabular}




\begin{tabular}{|c|c|c|}
\hline Artigo & Variáveis utilizadas & Trabalho de referência \\
\hline Damanpour (1996) & $\begin{array}{l}\text { Turbulência; variabilidade ambiental e da concorrência; diferentes con- } \\
\text { dições ambientais ou setores. }\end{array}$ & $\begin{array}{l}\text { Kim (1980); Zmud (1984); Aiken et al. (1980); } \\
\text { Damanpour (1987); Miller e Friesen (1983); } \\
\text { Kimberly e Evanisko (1981) }\end{array}$ \\
\hline Dickson e Weaver (1997) & $\begin{array}{l}\text { Dinamismo; ambiente externo; comportamento; incerteza geral; deman- } \\
\text { da tecnológica; volatilidade; previsibilidade dos mercados; potencial de } \\
\text { crescimento futuro; lucros; grau de internacionalização. }\end{array}$ & $\begin{array}{c}\text { Covin e Slevin (1989); Schultz, Slevin e Covin } \\
\text { (1995); Dickson e Weaver (1997) }\end{array}$ \\
\hline Chong e Chong (1997) & Ambiente industrial, econômico, tecnológico, competitivo; clientes. & - \\
\hline Kumar e Seth (1998) & $\begin{array}{l}\text { Políticas governamentais e regulamentadoras; clima competitivo; forne- } \\
\text { cedores; tecnologia; cliente. }\end{array}$ & Egelhoff (1988) \\
\hline Abramson e Ai (1998) & $\begin{array}{l}\text { Atitude local; cultura local; tecnologia; leis; ambiente; compromisso; } \\
\text { preço. }\end{array}$ & - \\
\hline Miller e Shamsie (1999) & Ambiente; organização; decisão. & Milliken (1987) \\
\hline Luo (1999) & Complexidade; dinamismo; hostilidade. & - \\
\hline Vickery, Calantone e Dröge (1999) & $\begin{array}{l}\text { Volatilidade nas práticas de marketing; taxa de obsolescência dos pro- } \\
\text { dutos; concorrentes; demanda e gostos; modos de produção ou serviço. }\end{array}$ & Miller e Dröge (1986) \\
\hline Buvik e John (2000) & $\begin{array}{l}\text { Fornecedor; especificidade de ativos; investimentos; faturamento bruto } \\
\text { anual do fabricante; volume anual comprado do fornecedor; relações de } \\
\text { longo prazo. }\end{array}$ & $\begin{array}{l}\text { Anderson (1985); Heide e John (1990); Noor- } \\
\text { dewier, João e Nevin (1990) }\end{array}$ \\
\hline Claycomb, Dröge e Germain (2001) & Demanda; produto; mudanças. & - \\
\hline $\begin{array}{l}\text { Waldman, Ramirez, House e Puranam } \\
(2001)\end{array}$ & Dinamismo; risco; expansão; estresse. & Khandwalla (1976) \\
\hline Sarkar, Echambadi e Harrison (2001) & Dinamismo tecnológico, competitivo e de mercado. & $\begin{array}{l}\text { Jaworski e Kohli (1993); Dickson e Weaver } \\
\text { (1997) }\end{array}$ \\
\hline Li e Atuahene-Gima (2002) & Preço; produto; tecnologia; competitividade. & Miller (1987) \\
\hline McGee e Sawyerr (2003) & $\begin{array}{l}\text { Variabilidade no ambiente; complexidade ambiental; importância do se- } \\
\text { tor. }\end{array}$ & $\begin{array}{c}\text { Duncan (1972); Daft, Sormunem, Parques } \\
(1988)\end{array}$ \\
\hline Hough e White (2004) & Ambientes dinâmicos ou estáticos. & Duncan (1972) \\
\hline Desarbo et al. (2004) & Tecnologia; mercado; competição. & - \\
\hline Lee, Lin e Pai (2005) & Clientes; concorrentes; produtos; preços concorrentes. & Kearns e Lederer (1999), \\
\hline Jorissen et al. (2005) & $\begin{array}{l}\text { Concorrentes; fornecedores; clientes; público; tecnologia; mercados fi- } \\
\text { nanceiros; governo; agências reguladoras; sindicatos. }\end{array}$ & - \\
\hline Babakus, Yavas e Haahti (2006) & Trabalho; fornecedores; clientes; capital. & - \\
\hline Lester et al. (2006) & Munificência; dinamismo; complexidade. & - \\
\hline
\end{tabular}




\begin{tabular}{|c|c|c|}
\hline Artigo & Variáveis utilizadas & Trabalho de referência \\
\hline Fink, Edelman e Hatten (2006) & Incerteza tecnológica. & Withey, Daft e Cooper (1983) \\
\hline Carmeli e Tishler (2006) & $\begin{array}{l}\text { Práticas comerciais; obsolescência dos produtos; ação dos concorrentes; } \\
\text { gostos e previsão de demanda; taxa de mudança da tecnologia de pro- } \\
\text { dução/serviço. }\end{array}$ & Miller e Dröge (1986) \\
\hline Agle et al. (2006) & Volatilidade do retorno das ações. & $\begin{array}{l}\text { Core Holt Hausen e Larcker (1999); Gray e Ca- } \\
\text { nella (1997); Miller, Wiseman, e Gomez-Mejia } \\
\text { (2002) }\end{array}$ \\
\hline $\begin{array}{l}\text { Krishnan, Martin e Noorderhaven } \\
\text { (2006) }\end{array}$ & Instabilidade; imprevisibilidade. & $\begin{array}{c}\text { Bergh e Lawless (1998); Keats e Hitt (1988); } \\
\text { Glick, Ogilvie e Miller (1990); Wholey e Brittain } \\
\text { (1989) }\end{array}$ \\
\hline Li, Bingham e Umphress (2007) & $\begin{array}{l}\text { Preferência dos clientes; necessidades dos clientes; concorrentes; mer- } \\
\text { cado. }\end{array}$ & - \\
\hline Paulraj e Chen (2007) & $\begin{array}{l}\text { Fornecedores e necessidades da empresa; fornecedores e qualidade dos } \\
\text { materiais; inspeção dos materiais; rejeição de materiais. }\end{array}$ & - \\
\hline Agbejule e Burrowes (2007) & $\begin{array}{l}\text { Taxa de mudança; taxa de inovação em produtos/serviços e no processo; } \\
\text { taxa de mudança no gosto dos clientes e suas preferências. }\end{array}$ & Miller e Friesen (1983) \\
\hline Argyres, Bercovitz e Mayer (2007) & Grau de incerteza associado com o projeto. & - \\
\hline Liao e Tu (2007) & $\begin{array}{l}\text { Gostos e preferências da demanda; inovação de produtos do setor; ob- } \\
\text { solescência dos produtos; taxa de inovação tecnológica no processo pro- } \\
\text { dutivo. }\end{array}$ & - \\
\hline Hsu e Wang (2008) & Clientes; tecnologia; concorrentes. & $\begin{array}{c}\text { Miller e Friesen (1978, 1983); Eisenhardt } \\
\text { (1989); Daft (2003) }\end{array}$ \\
\hline Cannella Jr., Parke e Lee (2008) & Ausência de padrão; imprevisibilidade; mudança inesperada. & Dess e Beard (1984); Keats e Hitt (1988) \\
\hline Wallace et al. (2010) & $\begin{array}{l}\text { Práticas de marketing; obsolescência de produtos e serviços; ações dos } \\
\text { concorrentes; demanda e gostos dos consumidores; valores e crenças; } \\
\text { avanços tecnológicos na indústria. }\end{array}$ & - \\
\hline
\end{tabular}


Além dos trabalhos apresentados no Quadro 3, evidenciam-se também os trabalhos de: Koberg e Ungson (1987), Koberg (1987), Miller, Dröge e Toulouse (1988), Shrader, Mulford e Blackburn (1989), Schneider e Meyer (1991), Russell e Russell (1992), Alexander (1991), Lang, Calantone e Gudmundson (1997), Gosselin (2005), Moschuris (2007), Sahadev (2008), Sun, Hsu e Hwang (2009), Townsend, Yeniyurt e Talay (2009) e Semadeni e Anderson (2010), que não tiveram suas variáveis especificadas pelos autores.

Observou-se que, entre os trabalhos que não explicitaram os indicadores para mensuração da incerteza ambiental, alguns citaram o(s) trabalho(s) que serviu(ram) de referência para a coleta dos dados na sua pesquisa. Essas informações podem ser visualizadas no Quadro 4.

Quadro 4 - Trabalhos de referência utilizados pelos artigos que não citaram as variáveis de mensuração da incerteza ambiental

\begin{tabular}{|c|c|}
\hline Artigo & Trabalho de referência \\
\hline Koberg e Ungson (1987) & Thompson (1967) e Ducan (1972) \\
\hline Koberg (1987) & Ducan (1972) \\
\hline Miller, Dröge e Toulouse (1988) & Ducan (s.d.) e Bourgeois III (s.d.) \\
\hline Shrader, Mulford e Blackburn (1989) & Koberg (1987) e Zammuto (1983) \\
\hline Alexander (1991) & Gordon e Narayanan (1984) \\
\hline Gosselin (2005) & Celly e Frazier (1996) \\
\hline Sahadev (2008) &
\end{tabular}

Fonte: elaborado pelos autores (2014).

Além destes, os estudos de Schoorman, Bazerman e Atkin (1981), Milliken (1987), Kay e Diamantopoulos (1987), Reed e Lemark (1996), Bhattacharya, Krishnan e Mahajan (1998), Zhang e Doll (2001), Greve (2002), Jones e Ryan (2002), Kreiser e Marino (2002), Callanan (2004), Akhter e Robles (2006), Beugre, Acar e Braun (2006), Judge e Blocker (2008) e Gravier, Randall e Strutton (2008) não foram incluídos, por terem sua metodologia direcionada a estabelecer um ensaio teórico sobre o tema e por não se aterem especificamente a identificar formas de mensuração da incerteza ambiental.

Entre os trabalhos analisados, observou-se uma expressiva contribuição da pesquisa quantitativa sobre os demais estudos. Verificou-se que, do total de 97 artigos analisados, 83 valeram-se de investigações de ordem quantitativa, representando $85 \%$ dos estudos, enquanto o restante (14 trabalhos) realizou ensaios teóricos sobre o tema representando $15 \%$ da amostra pesquisada.

Buscando auxiliar futuras pesquisas sobre a temática incerteza ambiental, no decorrer das análises de cada artigo, foram identificadas terminologias vinculadas ao assunto, que poderão ser utilizadas em futuras indagações científicas como palavras-chave. Tal informação pode ser observada no Quadro 5. 
Quadro 5 - Relação de terminologias que referenciam o conteúdo de incerteza ambiental

\begin{tabular}{|c|c|c|}
\hline \multicolumn{2}{|c|}{ TERMINOLOGIAS } \\
\hline Environmental Uncertainty & Environmental Turbulence & Response Uncertainty \\
\hline Effect Uncertainty & Environmental Unpredictability & State Uncertainty \\
\hline Environment & Environmental Variables & Supply Uncertainty \\
\hline Environmental Context & Environmental Volatility & Uncertainty \\
\hline Environmental Decision & E.U. & Uncertainty Future \\
\hline Environmental Information & Perceived Environmental Uncertainty & Uncertainty Management \\
\hline Environmental Instability & Perceived Strategic Uncertainty & Uncertainty Modeling \\
\hline Environmental Risk & Perceived Uncertainty & \\
\hline Environmental Scanning & P.E.U. & \\
\hline
\end{tabular}

Source: Authors (2014)

No intuito de identificar os trabalhos utilizados para fundamentar as pesquisas, foram selecionadas as referências utilizadas em cada um dos artigos. Assim, no Quadro 6, foram elencados os dez autores mais referenciados nas pesquisas e identificada a quantidade de vezes que foram citados. Além desta informação, apresenta-se o trabalho mais referenciado e o número de vezes que foi citado.

Observa-se que, embora Miller tenha sido o autor mais referenciado, o trabalho de Ducan (1972) foi consistentemente o trabalho mais mencionado nas pesquisas realizadas entre 1975 e 2010.

Quadro 6 - Referências mais citadas

\begin{tabular}{|c|c|l|c|}
\hline Autor & Cit. & \multicolumn{1}{|c|}{ Trabalho com maior número de citações } & Cit. \\
\hline MILLER, Dany & 65 & $\begin{array}{l}\text { MILLER, D.; FRIESEN, P. H. Strategy-making and environment: the third } \\
\text { link. Strategic Management Journal, v. 4, n. 3, p. 221-235, 1983. }\end{array}$ & 9 \\
\hline DUCAN, Robert B. & 57 & $\begin{array}{l}\text { DUNCAN, R. B. Characteristics of organizational environments and per- } \\
\text { ceived environmental uncertainty. Administrative Science Quarterly, } \\
\text { V. 17, n. 3, p. 313-327, 1972. }\end{array}$ & 49 \\
\hline PFEFFER, Jeffrey & 51 & $\begin{array}{l}\text { PFEFFER, J.; SALANCIK, G. R. The External Control of Organizations: } \\
\text { a resource dependence perspective. New York: Harper \& Row Pu- } \\
\text { blishers, 1978. }\end{array}$ & 26 \\
\hline HAMBRICK, Donald C. & 50 & $\begin{array}{l}\text { HAMBRICK, D. C. Environmental scanning and organizational strategy. } \\
\text { Strategic Management Journal, v. 3, n. 2, p. 159-174, 1982. }\end{array}$ & 10 \\
\hline LAWRENCE, Paul R. & 49 & $\begin{array}{l}\text { LAWRENCE, P. R.; LORSCH, J. W. Organization and Environment, Har- } \\
\text { vard University Press, Cambridge, MA, 1967. }\end{array}$ & 27 \\
\hline THOMPSON, James D. & 43 & $\begin{array}{l}\text { THOMPSON, J. D. Organizations in action. New York: McGraw-Hill, } \\
\text { 1967. }\end{array}$ & 39 \\
\hline MILES, Raymond E. & 42 & $\begin{array}{l}\text { MILES, R. E.; SNOW, C. C. Organizational strategy, structure, and pro- } \\
\text { cess. New York: McGraw-Hill. 1978. }\end{array}$ & 25 \\
\hline BOURGEOIS, L. J. III & 35 & $\begin{array}{l}\text { BOURGEOIS, L. J. III. Strategy and environment: a conceptual integra- } \\
\text { tion. Academy of Management Review, v. 5, n. 1, p. 25-39, 1980. }\end{array}$ & 10 \\
\hline DOWNEY, H. Kirk & 35 & $\begin{array}{l}\text { DOWNEY, H. K.; HELLRIEGEL, D.; SLOCUM, J. W. Jr. Environmental un- } \\
\text { certainty: the construct and its applications. Administrative Science } \\
\text { Quarterly, v. 20, p. 613-629, 1975. }\end{array}$ & 15 \\
\hline PORTER, Michael E. & 31 & $\begin{array}{l}\text { PORTER, M. E. Competitive strategy: techniques for analyzing indus- } \\
\text { tries and competitors. New York, NY: The Free Press, 1980. }\end{array}$ & 9 \\
\hline
\end{tabular}

Fonte: elaborado pelos autores (2014). 
Identificou-se que muitos trabalhos apresentaram como complemento ao estudo os instrumentos utilizados na pesquisa (questionários ou procedimentos detalhados de pesquisa) que passaram pelo crivo daquele estudo.

Essas pesquisas estão destacadas no Quadro 7. Ressalta-se que esses trabalhos podem contribuir para futuras pesquisas como exemplos de mecanismos para coleta de dados no que tange ao tema incerteza ambiental.

Quadro 7 - Trabalhos que apresentam instrumento ou procedimentos detalhados de pesquisa

\begin{tabular}{|c|c|c|}
\hline \multicolumn{2}{|c|}{ ARTICLES } \\
\hline Lorenzi, Jr and Jr (1981) & Miller (1993) & Abramson and Ai (1998) \\
\hline Miller and Dröger (1986) & $\begin{array}{c}\text { Germain, Dröge and Daugher- } \\
\text { ty (1994) }\end{array}$ & $\begin{array}{c}\text { Waldman, Ramírez, House and Puranam } \\
\text { (2001) }\end{array}$ \\
\hline McCabe (1987) & Tan and Litschert (1994) & $\begin{array}{c}\text { Desarbo, Benedetto, Song and Sinha } \\
\text { (2005) }\end{array}$ \\
\hline Koberg (1987) & Ganesan (1994) & Lee, Lin and Pai (2005) \\
\hline $\begin{array}{c}\text { Swamidass and Newell } \\
\text { (1987) }\end{array}$ & $\begin{array}{c}\text { Kumar, Scheer and Steenkamp } \\
(1995)\end{array}$ & Agbejule and Burrowes (2007) \\
\hline Schneider and Meyer (1991) & Magaliso (1995) & Hsu and Wang (2008) \\
\hline Sabherwal and King (1992) & $\begin{array}{c}\text { Wener, Brouthers and Brou- } \\
\text { thers (1996) }\end{array}$ & Wallace, Little, Hill and Ridge (2010) \\
\hline Kren and Kern (1993) & Kumar and Seth (1998) & \\
\hline
\end{tabular}

Source: Authors (2014)

Assim, com base na revisão bibliográfica e nos procedimentos metodológicos anteriormente apresentados, além da análise bibliométrica sobre a temática incerteza ambiental, a seguir serão apresentadas as considerações finais e sugestões para trabalhos futuros.

\section{CONSIDERAÇÕES FINAIS}

No decorrer deste artigo, diversas análises foram realizadas no intuito de explorar o tema incerteza ambiental, tendo como método a bibliometria. A partir da análise de 97 publicações ao longo do período de 1975 a 2010, pode-se destacar algumas características dessas pesquisas.

Diante dos resultados encontrados, é lícito que existam diversas pequenas redes de relacionamento de pesquisadores interessados em publicações sobre incerteza ambiental; no entanto, ressalta-se o fato de não existir uma constância de publicações em periódicos internacionais com alto impacto por parte desses estudiosos. Essa mesma consideração também é válida em termos de parcerias entre instituições de ensino.

Sabe-se que, nesse cenário, deve-se considerar, também, a morosidade do processo de análise dos trabalhos por parte dos periódicos. Porém, essa possibilidade pode ser praticamente descartada, pois o estudo abrangeu o espaço temporal de 35 anos, o que rejeita tal justificativa de baixa publicação.

Entre os periódicos, observou-se que o Academy of Management Journal obteve destaque no número de artigos publicados, o que sugere o interesse por publicações direcionadas à incerteza ambiental. Essa informação é oportuna para pesquisadores que se dedicam a desenvolver estudos nessa linha.

Os anos de 1987 e 2006 receberam destaque por conta da expressividade no número de artigos publicados; por outro lado, é necessário destacar que o total de artigos foi de apenas 16 em âmbito internacional. Observa-se que esse número não é tão expressivo quando consideradas as sugestões para trabalhos futuros presentes nos estudos analisados. Essas contribuições apontam para um universo gigantesco de possibilidades de pesquisas. 
Com relação às variáveis utilizadas para a mensuração da incerteza ambiental, é conclusiva a importância do estudo de Ducan (1972), que serviu de referência à elaboração de muitos instrumentos para coleta de dados, além de fundamentar grande parte dos trabalhos.

Dessa forma, conclui-se, com este trabalho, que a incerteza ambiental no decorrer dos anos ganhou espaço, porém não adquiriu forma metodológica uniforme. $O$ espaço é caracterizado pelo número de publicações internacionais, que é restrito, mas existente. A ausência de uma forma definida de método, conceito e instrumento deve-se às diferentes características dos segmentos e das empresas estudadas, que foram correlacionadas às peculiaridades ambientais, resultando em um contínuo amoldamento procedimental a cada estudo.

A principal limitação deste estudo reside no fato de que a base de dados é limitada a alguns periódicos e a filtros metodológicos dos autores, o que pode acarretar em deixar às margens da coleta algum trabalho relevante.

Para trabalhos futuros, recomenda-se a ampliação deste estudo, identificando outras variáveis, que não puderam ser estudadas neste trabalho, como, por exemplo, o país de origem dos pesquisadores e o local de elaboração da pesquisa, além da aplicação de alguma técnica de análise multivariada, tal como a análise de correspondência múltipla, que poderá identificar os estudos que possuem correlação. Além disso, recomenda-se a realização de estudo similar nas bases de dados nacionais.

Por fim, procurou-se abordar alguns aspectos que, certamente, merecem atenção futura por parte dos pesquisadores e gestores das organizações. Dessa forma, o esforço realizado para apresentar alguns elementos importantes dos estudos sobre incerteza ambiental será mais uma contribuição na direção de discussões mais profundas acerca do assunto.

\section{REFERÊNCIAS}

ABRAMSON, N. R.; AI, J. X. Practising relationship marketing in Southeast Asia: reducing uncertainty and improving performance. Management International Review, v. 38, p. 113-143, 1998.

AGBEJULE, A.; BURROWES, A. Perceived environmental uncertainty, supply chain purchasing strategy, and use of MAS information: an empirical study of finnish firms. Managerial Auditing Journal, v. 22, n. 9, p. 913-927, 2007.

AGLE, B. R. et al. Does CEO charisma matter? An empirical analysis of the relationships among organizational performance, environmental uncertainty, and top management team perceptions of CEO charisma. Academy of Management Journal, v. 49, n. 1, p. 161-174, 2006.

AKHTER, S. H.; ROBLES, F. Leveraging internal competency and managing environmental uncertainty: propensity to collaborate in international markets. International Marketing Review, v. 1, n. 23, p. 98-115, 2006.

ALEXANDER, J. A. Adaptive change in corporate control practices. Academy of Management Journal, v. 34, n. 1, p. 162-193, 1991.

ARAUJO, W. T. et al. Meta-análise das dissertações do curso de mestrado ciência da informação UFPB: 1990-1999. Revista Informação e Sociedade: estudos. João Pessoa, v. 10, n. 1, 2000.

ARGYRES, N. S.; BERCOVITZ, J.; MAYER, K. J. Complementarity and evolution of contractual provisions: an empirical study of IT service contracts. Organization Science, v. 18, n. 1, p. 3-19, 2007. 
BABAKUS, E.; YAVAS, U.; HAAHTI, A. Perceived uncertainty, networking and export performance: a study of Nordic SMEs. European Business Review, v. 1, n. 8, p. 4-13, 2006.

BEUGRÉ, C. D.; ACAR, W.; BRAUN, W. Transformational leadership in organizations: an environmentinduced model. International Journal of Manpower, v. 27, n. 1, p. 52-62, 2006.

BHATTACHARYA, S.; KRISHNAN, V.; MAHAJAN, V. Managing new product definition in highly dynamic environments. Management Science, v. 44, n. 11, p. 50-64, 1998.

BOULTON, W. R. et al. Strategic planning: determining the impact of environmental characteristics and uncertainty. Academy of Management Journal, v. 25, n. 3, p. 500-509, 1982.

BOYD, B. Corporate linkages and organizational environment: a test of the resource dependence model. Strategic Management Journal, v. 11, p. 419-430, 1990.

BUVIK, A.; JOHN, G. When does vertical coordination improve industrial purchasing relationship? Journal of Marketing, v. 64, n. 4, p. 54-64, 2000.

BUCHKO, A. A. Conceptualization and measurement of environmental uncertainty: an assessment of the Miles and Snow perceived environmental uncertainty scale. Academy of Management Journal, v. 37, n. 2, p. 410-425, 1994.

CALLANAN, G. A. What would Machiavalli think? an overview of the leadership challenges in team-based structures. Team Performance Management, v. 3, n. 10, p. 77-83, 2004.

CANNELLAJR., A. A. C.; PARK, J-H.; LEE, H-U. Top management team functional background diversity and firm performance: examining the roles of team member collocation and environmental uncertainty. n. 4, v. 51, Academy of Management Journal, 768-784, 2008.

CARMELI, A.; TISHLER, A. The relative importance of the top management team's managerial managerial skills. International Journal of Manpower, v. 27, n. 1, p. 9-35, 2006.

CHONG, V. K.; CHONG, K. M. Strategic choices, environmental uncertainty and SBU performance: a note on the intervening role of management accounting systems. Accounting and Business Research, v. 27, n. 4, p. 268-276, 1997.

CHOW, C. W.; HADDAD, K. M. Relative performance evaluation and risk taking in delegated investment decisions. Decision Sciences, v. 22, n. 3, p. 583-593, 1991.

CLAYCOMB, C.; DRÖGE, C.; GERMAIN, R. Applied process knowledge and market performance: the moderating effect of environmental uncertainty. Journal of Knowledge Management, v. 5, n. 3, p. 264-277, 2001.

DAMANPOUR, F. Organizational complexity and innovation: developing and testing multiple contingency models. Management Science, v. 42, n. 5, p. 693-716, 1996.

DESARBO, W. S. et al. Revisiting the Miles and Snow strategic framework: uncovering interrelationships between strategic types, capabilities, environmental uncertainty, and firm performance. Strategic Management Journal, v. 26, p. 47-74, 2005.

DICKSON, P. H.; WEAVER, K. M. Environmental determinants and individual-level moderators off alliance use. Academy of Management Journal, v. 40, n. 2, p. 404-425, 1997. 
DUNCAN, R. B. Characteristics of organizational environments and perceived environmental uncertainty. Administrative Science Quarterly, v. 17, n. 3, p. 313-327, 1972.

FINK, R. C.; EDELMAN, L. F.; HATTEN, K. J. Relational exchange strategies, performance, uncertainty, and knowledge. Journal of Marketing Theory and Practice, v. 2, n. 14, p. 139-153, 2006.

FORD, J. D. Departartamental context and formal structure as constraints on leader behavior. Academy of Management Journal, v. 24, n. 2, p. 274-288, 1981.

GANESAN, S. Determinants of long-term orientantion in buyer-seller relationships. Journal of Marketing, v. 58, n. 2, p. 1-19, 1994.

GALASKIEWICZ, J.; SHATIN, D. Leadership and networking among neighborhood human service organizations. Administrative Science Quarterly, v. 26, p. 434-448, 1981.

GERLOFF, E. A.; MUIR, N. K.; BODENSTEINER, W. D. Three components of perceived environmental uncertainty: an exploratory analysis of the effects of aggregation. Journal of Management, v. 17, n. 4, p. 749-768, 1991.

GERMAIN, R.; DRÖGE, C.; DAUGHERTY, P. J. The effect of just-in-time selling on organization structure: an empirical investigation. Journal of Marketing Research, v. 31, n. 4, p. 471-483, 1994.

GOSSELIN, M. An empirical study of performance measurement in manufacturing firms. International Journal of Productivity and Performance Management, v. 5, n. 54, p. 419-437, 2005.

GRAVIER, M. J.; RANDALL, W. S.; STRUTTON, D. Investigating the role of knowledge in alliance performance. Journal of Knowledge Management, v. 12, n. 4, p. 117-130, 2008.

GREVE, H. R. Sticky aspirations: organizational time perspective and competitiveness. Organization Science, v. 13, n. 1, p. 1-17, 2002.

GUL, F. A. The effects of management accounting systems and environmental uncertainty on small business managers' performance. Accounting and Business Research, v. 22, n. 85, p. 57-61, 1991.

HANNAN, M. T.; FREEMAN, J. The population ecology of organizations. American Journal of Sociology, v. 82, n. 5, p. 929-964, 1977.

HITT, M. A.; IRELAND, R. D.; PALIA, K. A. Industrial firm's grand strategy and functional importance: moderating effects of technology and uncertainty. Academy of Management Journal, v. 25, n. 2, p. 265-298, 1982.

HOUGH, J. R.; WHITE, M. A. Scanning actions and environmental dynamism, gathering information for strategic decision making. Management Decision, v. 5, n. 42, p. 7981-793, 2004.

HSU, I-C.; WANG, Y-S. A model of intraorganizational knowledge sharing: development and initial test. Journal of Global Information Management, v. 16, n. 3, p. 45-73, 2008.

HUBER, G. P.; O'CONNEL, M. J.; CUMMINGS, L. L. Perceived environmental uncertainty: effects of information and structure Academy of Management Journal, v. 18, p. 725-740, 1975. 
BOURGEOIS III, L. J. Strategic goals, perceived uncertainty, and economic performance in volatile environments. Academy of Management Journal, v. 28, n. 3, p. 548-573, 1985.

IRELAND, R. D. et al. Strategy formulation processes: differences in perceptions of strength and weaknesses indicators and environmental uncertainty by managerial level. Strategic Management Journal, v. 8, p. 469-485, 1987.

JONES, R. B. Determinants of administrative intensity. Journal of Management, v. 3, n. 2, p. 1523, 1977.

JONES, R. T.; RYAN, C. Matching process choice and uncertainty, modeling quality management. Business Process Management Journal, v. 8, n. 2, p. 161-168, 2002.

JORISSEN, A. et al. Real versus sample-based differences in comparative family business research. Family Business Review, v. 3, n. 18, p. 229-246, 2005.

JUDGE, W. Q.; BLOCKER, C. P. Organization capacity for change and strategic ambidexterity: flying the plane while rewiring it. European Journal of Marketing, v. 42, n. 9/10, p. 915-916, 2008.

KAY, N. M.; DIAMANTOPOULOS, A. Uncertainty and synergy: towards a formal model of corporate strategy. Managerial and Decision Economics, v. 8, p. 121-130, 1987.

KUMAR, N.; SCHEER, L. K.; STEENKAMP, J-B E. M. The effects of supplier fairness on vulnerable resellers. Journal of Marketing Research, v. 32, n. 1, p. 54-65, 1995.

KOBERG, C. S. Resource scarcity, environmental uncertainty, and adaptive organizational behavior. Academy of Management Journal, v. 30, n. 4, p. 798-807, 1987.

; UNGSON, G. R. The effects of environmental uncertainty and dependence on organizational structure and performance: a comparative study. Journal of Management, v. 13, n. 4, p. 725-737, 1987.

KOPP, D. G.; LITSCHERT, R. J. A buffering response in light of variation in core technology, perceived environmental uncertainty, and size. Academy of Management Journal, v. 23, n. 2, p. 252-266, 1980.

KREISER, P.; MARINO, L. Analyzing the historical development of the environmental uncertainty construct. Management Decision, v. 9, n. 40, p. 895-905, 2002.

KREN, L.; KERR, J. L. The effects of behaviour monitoring and uncertainty on the use of performancecontingent compensation. Accounting and Business Research, v. 23, n. 90, p. 159-168, 1993.

KRISHNAN, R.; MARTIN, X.; NORRDERHAVEN, N. G.; When does trust matter to alliance performance? Academy of Management Journal, v. 49, n. 5, p. 894-917, 2006.

KUMAR, S.; SETH, A. The design of coordination and control mechanisms for managing join venture-parent relationships. Strategic Management Journal, v. 19, n. 6, p. 579-599, 1998.

LEBLEBICI, H.; SALANICIK, G. R. Stability interorganizational exchanges: rulemaking processes of the Chicago board of trade. Administrative Science Quarterly, v. 27, p. 227-242, 1982.

LEE, G-G.; LIN, H-F.; PAI, J-C. Influence of environmental and organization factors on the success of internet-based interorganizational systems planning. Internet Research, v. 5, n. 15, p. 527-543, 2005. 
LESTER, R. H. et al. Initial public offering investor valuations: an examination of top management team prestige and environmental uncertainty. Journal of Small Business Management, v. 1, n. 44, p. 1-26, 2006.

LEV, B. Environmental uncertainty reduction by smoothing and buffering: an empirical verification. Academy of Management Journal, v. 18, p. 864-871, 1975.

LI, H.; ATUAHENE-GIMA, K. The adoption of agency business activity, product innovation, and performance in Chinese technology ventures. Strategic Management Journal, v. 23, n. 6, p. 469490, 2002.

; BINGHAM, J. B.; UMPHRESS, E. E. Fairness from the top: perceived procedural justice and collaborative problem solving in new product development. Organization Science, v. 18, n. 2, p. 200-216, 2007.

LIAO, K.; TU, Q.; Leveraging automation and integration to improve manufacturing performance under uncertainty: an empirical study. Journal of Manufacturing Technology Management, v. 19, n. 1, p. 38-51, 2007.

LORENZI, P.; JR., H. P. S.; JR., J. W. S. Perceived environmental uncertainty: an individual or environmental attribute? Journal of Management, v. 7, n. 2, p. 27-41, 1981.

LUO, Y. Environment-strategy-performance relations in small business in China: a case of township and village enterprises in southern China. Journal of Small Business Management, v. 37, n. 1, p. 37-52, 1999.

MCCABE, D. L. Buying group structure: constriction at the top. Journal of Marketing, v. 51, p. 8998, 1987.

MCGEE, J. E.; SAWYERR, O. O. Uncertainty and information search activities: a study of ownermanagers of small high-technology manufacturing firms. Journal Small Business Management, v. 4, n. 41, p. 385-401, 2003.

MANGALISO, M. P. The strategic usefulness of management information as perceived by middle managers. Journal of Management, v. 21, n. 2, p. 231-250, 1995.

MATTHEWS, C. H.; SCOTT, S. G. Uncertainty and planning in small and entrepreneurial firms: an empirical assessment. Journal of Small Business Management, v. 33, n. 4, p. 34-52, 1995.

MILLER, D. The structural and environmental correlates of business strategy. Strategic Management Journal, v. 8, p. 55-76, 1987.

MILES, R. E.; SNOW, C. C.; PFEFFER, J. Organization-environment: concepts and issues. Industrial Relations: a journal of economy and society, v. 13, n. 3, p. 244-264, 1974.

MILLER, D. Enviromental fit and internal fit. Organization Science, v. 3, n. 2, May 1992. Industry and country effects on managers perceptions of environmental uncertainties. Journal of International Business Studies, v. 24, n. 4, p. 693-714, 1993.

; DRÖGE, C. Psychological and traditional determinants of structure. Administrative Science Quarterly, v. 31, p. 539-560, 1986. 
; DRÖGE, C.; TOULOUSE, J. Strategic process and content as mediators between organizational context and structure. Academy of Management Journal, v. 31, n. 3, p. 544-569, 1988.

; FRIESEN, P. H. Strategy-making and environment: the third link. Strategic Management Journal, v. 4, n. 3, p. 221-235, 1983.

; SHAMSIE, J. Strategic responses to three kinds of uncertainty: product line simplicity at the Hollywood film studios. Journal of Management, v. 25, n. 1, p. 97-116, 1999.

MILLIKEN, F. J. Three types of perceived uncertainty about the environment: state, effect, and response uncertainty. The Academy of Management Review, v. 12, n. 1, p. 133-143, 1987.

MOSCHURIS, S. J. Triggering mechanisms in make-or-buy decisions: an empirical analysis. Journal of Supply Chain Management, v. 43, n. 1, p. 40-49, 2007.

PAULRAJ, A.; CHEN, I. J. Environmental uncertainty and strategic supply management: a resource dependence perspective and performance implications. Journal of Supply Chain Management, v. 43, n. 3, p. 29-42, 2007.

REED, R.; LEMAK, D. J.; MONTGOMERY, J. C. Beyond process: TQM content and firm performance. Academy of Management, v. 21, n. 1, p. 173-202, 1996.

RUSSELL, R. D.; RUSSELL, C. J. An examination of the effects of organizational norms, organizational structure, and environmental uncertainty on entrepreneurial strategy. Journal of Management, v. 18, n. 4, p. 639-565, 1992.

SABHERWAL, R.; KING, W. R. Decision processes for developing strategic applications of information systems: a contingency approach. Decision Sciences, v. 23, n. 4, p. 917-943, 1992.

SABHERWAL, R.; KIRS, P. The alignment between organizational critical success factors and information technology capability in academic institutions. Decision Sciences, v.25, n.2, 301-330, 1994.

SAHADEV, S. Economic satisfaction and relationship commitment in channels: the moderating role of environmental uncertainty, collaborative communication and coordination strategy. European Journal of Marketing, v. 42, n. 1/2, p. 178-195, 2008.

SARKAR, M. B.; ECHAMBADI, R.; HARRISON, J. S. Alliance entrepreneurship and firm market performance. Strategic Management Journal, v. 22, p. 701-711, 2001.

SAWYERR, O. O. Environmental uncertainty and environmental scanning activities of Nigerian manufacturing executives: a comparative analysis. Strategic Management Journal, v. 14, n. 4, p. 287-299, 1993.

SCHNEIDER, S. C.; MEYER, A. de. Interpreting and responding to strategic issues: the impact of national culture. Strategic Management Journal, v. 12, n. 4, p. 307-320, 1991.

SCHOORMAN, F. D.; BAZERMAN, M. H.; ATKIN, R. S. Interlocking directorates: a strategy for reducing environmental uncertainty. The Academy of Management Review, v. 6, n. 2, p. 243$251,1981$. 
SEMADENI, M.; ANDERSON, B. S. The followers dilemma: innovation and imitation in the professional services industry. Academy of Management Journal, v. 53, n. 5, p. 1.175-1.193, 2010.

SHRADER, C. B.; MULFORD, C. L.; BLACKBURN, V. L. Strategic and operational planning, uncertainty, and performance in small firms. Journal of Small Business Management, v. 27, n. 4, p. 45-60, 1989.

SILVEIRA-MARTINS, E. et al. Incerteza ambiental: um estudo bibliométrico em bases de dados nacionais. Revista da Faculdade de Administração e Economia, v. 4, n. 2, p. 141-157, 2013.

; TAVARES, P. M. Associação entre capacidades gerenciais e desempenho mediadas pela incerteza ambiental: um estudo na agrox. Revista Cesumar - Ciências Humanas e Sociais Aplicadas, v. 19, n. 2, p. 347-370, 2014a.

; TAVARES, P. M. Processo de formulação de estratégias: capacidade mercadológica, incerteza ambiental e desempenho. Organizações em Contexto, v. 10, n. 20, p. 297-322, 2014b.

SIMON, H. A. Organizations and markets. Journal of Economic Perspectives, v. 5, n. 2, p. 25-44, 1991.

Rational decision-making in business organizations. Economic Sciences, Nobel Memorial Lecture, p. 343-371, 1978.

STEARNS, T. M.; HOFFMAN, A. N.; HEIDE, J. B. Performance of commercial television stations as an outcome of interorganizational linkages and environmental conditions. Academy of Management Journal, v. 30, n. 1, p. 71-90, 1987.

SUN, S-Y.; HSU, M-H.; Hwang, W-J. The impact of alignment between supply chain strategy and environmental uncertainty on SCM performance. Supply Chain Management: a International Journal, v. 14, n. 3, p. 201-212, 2009.

SWAMIDASS, P. M.; NEWEEL, W. T. Manufacturing strategy, environmental uncertainty and performance: a path analytic model. Management Science, v. 33, n. 4, p. 509-524, 1987.

TAN, J. J.; LITSCHERT, R. J. Environment-strategy relationship and ITS performance implications: an empirical study of the Chinese electronics industry. Strategic Management Journal, v. 15, $n$. 1, p. 1-20, 1994.

TOWNSEND, J. D.; YENIYURT, S.; TALAY, M. B. Getting to global: an evolutionary perspective of brand expansion in international markets. Journal of International Business Studies, v. 40, p. 539-558, 2009.

TUSHMAN, M. L.; ANDERSON, P. Technological discontinuities and organizational environments. Administrative Science Quarterly, v. 31, p. 439-465, 1986.

UMANATH, N. S.; RAY, M. R.; CAMPBELL, T. L. The impact of perceived environmental uncertainty and perceived agent effectiveness on the composition of compensation contracts. Management Science, v. 39, n. 1, p. 32-45, 1993.

VICKERY, S.; CALATONE, R.; DRÖGE, C. Supply chain flexibility: an empirical study. Journal of Supply Chain Management, v. 35, n. 3, p. 16-24, 1999. 
WALDMAN, D. A. et al. Does leadership matter? CEO leadership attributes and profitability under conditions of perceived environmental uncertainty. Academy of Management Journal, v. 44, $n$. 1, p. 134-143, 2001.

WALLACE, J. C. et al. CEO regulatory foci, environmental dynamism, and small firm performance. Journal of Small Business Management, v. 48, p. 4, p. 580-604, 2010.

WEED, S. E.; MITCHELL, T. R. The role of environmental and behavioral uncertainty as a mediator of situation - performance relationships. Academy of Management Journal, v. 23, n. 1, p. 38-60, 1980.

WERNER, S.; BROUTHERS, L. E.; BROUTHERS, K. D. International risk and perceived environmental uncertainty: the dimensionality and internal consistency of Miller's measure. Journal of International Business Studies, v. 27, n. 3, p. 571-587, 1996.

ZHANG, Q.; DOLL, Q. F. The fuzzy front end and success of new product development: a causal model. European Journal of Innovation Management, v. 4, n. 2, p. 95-112, 2001. 